\title{
When COVID-19 delays the management of an urgent heart condition: rare case of a Spontaneous Dissection of two Coronary Arteries.
}

\author{
Gislain Endamena ${ }^{1}$, Mazou Temgoua ${ }^{2}$, Sylvain Chanseaume $^{1}$, Enver Hilic ${ }^{1}$, Lise Camus ${ }^{1}$, \\ Alexandra Chanseaume ${ }^{1}$, Alexandru Mischie ${ }^{1}$, Kane Karamoko ${ }^{1}$, Nouhoun Diallo ${ }^{1}$, Sami \\ Assi $^{1}$, and Romain Eschalier ${ }^{3}$ \\ ${ }^{1}$ Hospital Centre Montlucon \\ ${ }^{2}$ University of Yaounde I \\ ${ }^{3} \mathrm{CHU}$ Clermont-Ferrand
}

May 11, 2021

\begin{abstract}
COVID-19 is a worldwide crisis with a great impact in health structures. Delay in the management of routine medical conditions has been reported during this pandemic. We describe the case of a spontaneous dissection coronary arteries which has been initially misclassified as a case of COVID-19 infection
\end{abstract}

\section{Introduction}

Spontaneous Coronary Artery Dissection (SCAD) is a rare cause of acute coronary syndrome (ACS) and sudden death. It is defined as a separation within the coronary artery wall secondary to intramural hemorrhage, with or without tearing of the intima, thus modifying arterial architecture with the creation of a true and false channels. The starting point is often an intimal breach or even a hemorrhage of the vasavasorum [1]. SCAD preferentially affects the young population with almost no traditional cardiovascular risk factors and especially female with a sex ratio $\mathrm{M} / \mathrm{F}$ of $1 / 5$ [2]. It is responsible for 0.1 to $0.4 \%$ of all acute coronary syndromes [3]. It is an important cause of ACS in women and represents approximately $25 \%$ of acute coronary syndromes in women under 50 years old [4]. It has the same clinical presentation as acute coronary syndromes secondary to plaque ruptures. Many triggering factors are mentioned in the occurrence of a SCAD including physical or emotional stress [5].

Currently, the whole world experiences a very stressful condition generated by COVID-19 pandemic. Since the beginning of this pandemic, health care facilities precisely emergency room are overcrowded. So, with that massive influx of patients, many other diseases are misdiagnosed [6]. Xiang et al. in their study analysing the management and outcomes of patients with ST segment elevation myocardial infarction during the COVID-19 pandemic in China found an important decrease of the hospitalized patient with ST segment elevation myocardial infarction still the outbreak of COVID 19 pandemic. Also, they found a delay in management that patients according to the reperfusion strategy [6]. Similarly, Rashid et al . found a decline of acute myocardial infarction related hospitalization associated with a raise in the number of cases of out-of-hospital cardiac arrest since the beginning of this pandemic, particularly after the implementation of social confinement measures during the COVID-19 outbreak in England. Consequently, the reorganization of hospital services and staff in response to COVID-19 outbreak affected routine management of other diseases 
[7]. Through this case, we wanted to show the harmful influence of the current pandemic in the management of potential life-threatening issue as SCAD.

\section{Case presentation}

This is a 61-year-old patient, an active smoker at 20 pack-years, in full professional activity during the period of total confinement, has presented a presyncope in the morning around $09.50 \mathrm{am}$ without chest pain, or loss of consciousness or fall with notion of chills a few hours earlier. She was transported by the firefighters at 10:30 am for the Montluçon Hospital Center. Patient was received around $11.40 \mathrm{am}$ and referred to its sector hospital (Gueret Hospital Center) in accordance with the COVID-19 Plan from the Regional Health Agency for screening as suspected of Coronavirus infection 19. On arrival at the emergency room of this health facility, an electrocardiogram carried out revealed an apicolateral and inferior persistent ST segment elevation with mirror image on anterior area (figure 1). Then, patient has been transported to the initial center for emergency coronary angiography where she arrived at 3:30 p.m. Coronary angiography was performed and revealed the presence of a thrombus in the distal anterior interventricular artery with the appearance of "stick insect", a thrombus on the dominant circumflex artery obstructing the second marginal with a "radish tail" aspect and a right network free from any atheromatous lesion (Figures 2). Transthoracic cardiac ultrasound found good biventricular systolo-diastolic function with a left ventricular ejection fraction of $69 \%$, no abnormalities in segmental and global kinetics of the left ventricle. Troponin I was $2465 \mathrm{pg} / \mathrm{ml}$, Creatine phosphokinase at $1874 \mathrm{pg} / \mathrm{ml}$, C-reactive protein $1.1 \mathrm{mg} / \mathrm{l}$, leukocytes at $13,81.10^{9}$ /l. The medical treatment included glycoprotein IIb / IIIa inhibitor, double anti-aggregation platelet and anticoagulant (unfractionated heparin), beta-blocker (bisoprolol) on Day 1 hospitalization. Bisoprolol has been changed to ivabradine because the patient remained tachycardic with low blood pressure. We noted on the Day 3 electrocardiogram, presence of a $\mathrm{Q}$ wave of apico-lateral and inferior necrosis with persistence of the elevation from the same territory. Treatment with acetylsalicylic acid, clopidogrel, ivabradine, inhibitor of the proton pump was given as an exit treatment. Magnetic resonance imaging (MRI) was performed on an outpatient basis at one month and found: a sequelae of infero-latero-moderate, infero-septo-average, lateroapical, infero-apical and septo-apical with thinning of the middle and apical walls measuring respectively $3 \mathrm{~mm}$ and $4 \mathrm{~mm}$; a left ventricular ejection fraction of $47 \%$.

Coronarography control with optical coherence tomography (OCT) performed two months later allowed to highlight a complete healing of the lesions (Figure 3). OCT does not highlighted the intimal breach (figure 4).

\section{Discussion}

Formerly assimilated to a classic acute coronary syndrome, the diagnosis of SCAD was based mainly on the presence of an intimal radiolucent flap associated with a contrast in the arterial wall on coronary angiography. Currently, studies on SCAD permit to establish a classification into 3 angiographic types [8]: Type 1 (representing the classic description with intimal flap), type 2 (extensive and diffuse tubular lesions with a plane of dissection not visible which may result in complete coronary occlusion), type 3 (multiple focal tubular lesions due to intramural hematoma mimicking atherosclerosis).

Our patient presented a feature of a double dissection with a type 1 lesion on the distal anterior interventricular artery and a type 2 lesion on the second left marginal. Type 2, sometimes resulting in compression of the vascular lumen due to intramural hematoma without intimal flap, it is the most frequently encountered in the different series representing $67 \%$ of cases. Type 1 follow-up with an intimal flap which represents $29 \%$ of cases. Type 3 mimicking atherosclerotic lesions is only found in $4 \%$ of cases [8]. The descending anterior interventricular artery is usually the most affected during SCAD. However, polyarterial lesions could be found in a range of $20-25 \%$ of cases [9].

Patients with SCAD generally have arterial fragility without atheroma or calcifications which may limit the progression of coronary dissection. This fragility can be acquired during pregnancy, oral contraception, hormone therapy, systemic inflammatory diseases; congenital condition as in fibromuscular dysplasia, Marfan disease, syndrome Ehlers-Danlos, connectivitis, or even idiopathic [10, 11]. In the series by Saw etal asses- 
sing the baseline characteristics, predisposing factors and clinical outcomes of 168 patients with a SCAD, the association with a detailed screening of other non-coronary arterial disease had revealed that $72 \%$ of these patients also presented with fibromuscular dysplasia [5]. However, our patient did not present clinical elements that could suggest any of the above conditions cited. These changes in the arterial wall are generally not sufficient to explain SCAD. This pathology appears to be multifactorial. An extrinsic trigger appears essential, such as emotional stress, intense physical exercise, or maneuvers such as Valsalva [10, 11]. This is also mentioned in the series by Saw et al . In this study physical or emotional stress was implicated as triggering factors in $56 \%$ of cases [5]. Likewise, in our patient we could clearly evoke an emotional and important professional stress. In fact, concomitantly with the occurrence of this case of spontaneous dissection of two coronary arteries, we were living a period of general confinement in France due to the COVID-19 pandemic. Very anxiety-provoking situation both professional and personal for our patient who continue to carry out her professional activities despite the high risk of contracting a particularly fatal viral disease. We therefore think that the strong stress at the same time emotional, physical, and professional would be the triggering factor. The clinical presentation of a SCAD is similar to that of an acute coronary syndrome by rupture atherosclerotic plaque. In more than half of the cases, we note electrocardiographic changes as ST segment elevation associated with elevation of enzymes myocardial (troponin). The rest of the cases will present as acute non-ST elevation coronary syndromes.

Some may also present with short term life-threatening ventricular arrhythmias and others still in cardiac arrest. In our case, we had a typical electrocardiographic presentation of a myocardial infarction with inferolateral ST segment elevation and an anterior mirror image. Of course, we had an enzymatic movement with elevation of troponins I. In the study done by the Mayo Clinic in the United States of America with the highest number of patients with spontaneous coronary arteries dissection, aimed at determining the prevalence of coronary tortuosity in these patients, it appears that tortuosity of coronary arteries is a risk factor for SCAD and even recurrence [12].

As part of the SCAD, coronary angiography remains the first-line examination and sometimes allows to visualize thrombi in the real channel, but it makes it difficult to visualize hematoma of the artery wall. It also helps to determine the type of lesion. It is therefore systematically recommended an association with intravascular imaging as Optical Coherence Tomography

(OCT) or intravascular ultrasonography (IVUS). The optical coherence tomography has better resolution than intravacular ultrasonography. It allows to confirm the guide's position in the real channel, detail the site of the wall hematoma and intimal breach; to facilitate an accurate assessment of the size of the artery and optimize stent expansion in the event of underlying angioplasty [13]. However, OCT therefore allows a detailed overview of the arterial anatomy of patients [14]. This technique is limited by its lack of visualizing deep layers of the arterial wall [14]. Compared to intravascular ultrasound, OCT is clearly superior in terms of the location of the initial intimal lesion and in the exploration of the intimo-medial membrane (intimal flap) $[14,15]$.

The treatment of SCAD could be medical with or without stenting and the prognostic is generally good [13]. Our treatment consists of an antiplatelet drug, a beta-blocker in order to reduce the pressure shearing action on the arterial wall and thus stop intramural bleeding favorizing the repair of intimal breach. The myocardial sequelae were found after one month in our patient probably because of the association of two coronary arteries involvement and the delay of management. Although these sequelae have disappeared after two months with good medical therapy.

\section{Conclusion}

Spontaneous dissection of coronary arteries is a rare cause of acute coronary syndrome. As for takotsubo cardiomyopathy, it should always be evoked in young female with thoracic pain in the context of severe stress. The health practitioners should be more alerted specially during this current pandemic.

Acknowledgments: We acknowledge the patient who have accepted to participate in this study, and also all the administrative staff of hospital center of Montlucon. 


\section{Abbreviations}

ACS: acute coronary syndrome

COVID-19: Coronavirus infection disease 2019

CPK: Creatine phosphokinase

CRP: $\mathrm{C}$ reactive protein

$\mathrm{Mg} / \mathrm{l}:$ milligram per liter

$\mathrm{Mg} / \mathrm{ml}$ : picogram per milliliter

PCR: polymerase chain reaction

SARS COV 2: Severe acute respiratory syndrome Coronavirus 2

SCAD: Spontaneous dissection of coronary arteries

\section{Diclosures}

Approval of the research protocol: Formal ethical approval from the University Research Ethics Board was not required for the completion of this study.

Informed consent: Written informed consent for publication of this case report was obtained from the patient.

Data Availability Statement : The data that support the findings of this study are available from the corresponding author upon reasonable request.

Registry and registration no. of the study : N/A.

Animal studies: N/A.

Conflict of interest: None

Funding: None

\section{Author Contribution :}

Management of the case : All the authors

Manuscript writing : GBE

Critical revision : MNT

Supervision : RE

\section{References}

1) Berrada N.E.M., Zaimi A., Ezzouak A., et al. Post-partum acute coronary syndrome secondary to spontaneous coronary dissection: report of a case. Pan Afr Med J. 2015; 20: 249

2) Tweet M. S., Hayes S. N., Pitta S. R., et al. Clinical features, management and prognosis of spontaneous coronary artery dissection. Circulation, 2012, July 31. 126 (5): 579-88

3) Mortensen K. H., Thuesen L., Kristensen I. B. Spontaneous coronary artery dissection: a Western Denmark Heart Registry study. Catheter Cardiovasc Interv, 2009, Nov 1. 74 (5): 710-7

4) Saw J., Aymong E., Mancini G. B., et al. Nonatherosclerotic coronary artery disease in young women. Can J Cardiol, 2014, July 30 (7): 814-9 
5) Saw J., Aymong E., Sedlak T. et al. Spontaneous coronary artery dissection: association with predisposing arteriopathies and precipitating stressors and cardiovascular outcomes. Circ Cardiovasc Interv, 2014, Oct. 7 (5): $645-55$

6) Xiang D., Xiang X., Zhang W. et al. Management and Outcomes of Patients With STEMI During the COVID-19 Pandemic in China. J A C C. 2020, Sept 15 (10) : $1318-24$

7) Rashid M., Gale C.P., Curzen N., et al. Impact of Coronavirus Disease 2019. Pandemic on the Incidence and Management of Out-of-Hospital Cardiac Arrest in Patients Presenting With Acute Myocardial Infarction in England. J Am Heart Assoc. 2020; 9: e018379

8) Tweet M. S., Eleid M. F., Best P. J., et al. Spontaneous coronary artery dissection: revascularization versus conservative therapy. Circ Cardiovasc Interv, 2014, Dec 7 (6): 777-86

9) Shahid A. Spontaneous coronary artery dissection, Vol. 14, N³8 - 22 Feb 2017

10) Saw J., Mancini G.J.B., Humphries KH. Contemporary review on spontaneous coronary artery dissection. JACC2016; 68: 297-312

11) Yip A, Saw J. Spontaneous coronary artery dissection - a review. Cardiovasc Diagn Ther 2015; 5: 37-48

12) Eleid M.F., Guddeti R.R., Tweet M.S., et al. Coronary artery tortuosity in spontaneous coronary artery dissection: angiographic characteristics and clinical implications. Circ Cardiovasc Interv 2014; 7: 656- 62

13) Alfonso F., Bastante T., Rivero F. Spontaneous coronary artery dissection. Circ J, 2014. 78 (9): 2099-110

14) Alfonso F., Paulo M., Gonzalo N., et al. Diagnosis of spontaneous coronary artery dissection by optical coherence tomography. J Am Coll Cardiol 2012; 59: 1073-9

15) Paulo M., Sandoval J., Lennie V., et al. Combined use of OCT and IVUS in spontaneous coronary artery dissection. JACC Cardiovasc Imaging 2013; 6: 830-2

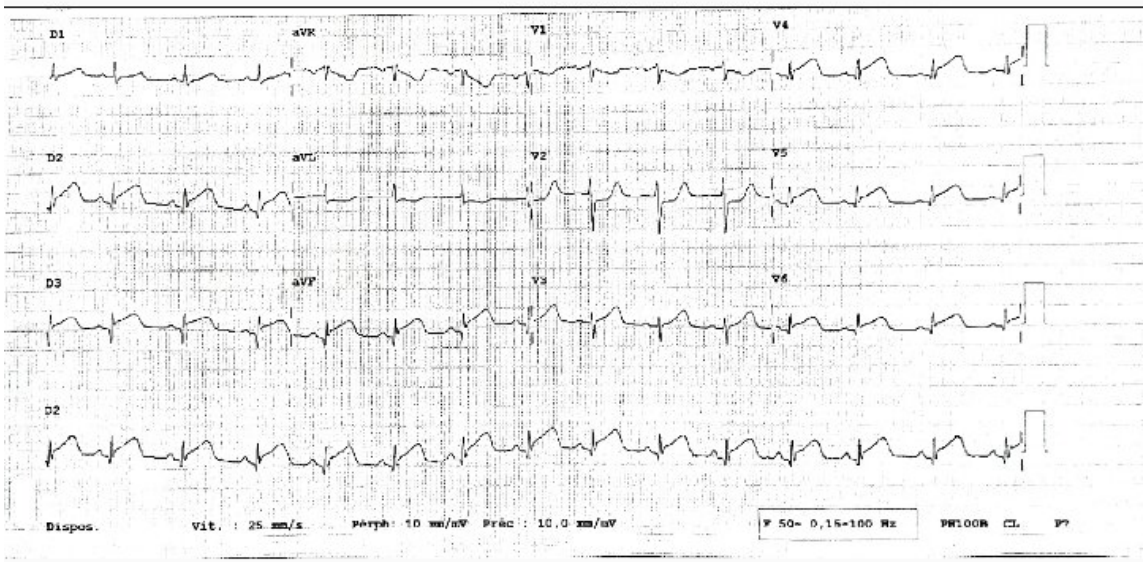




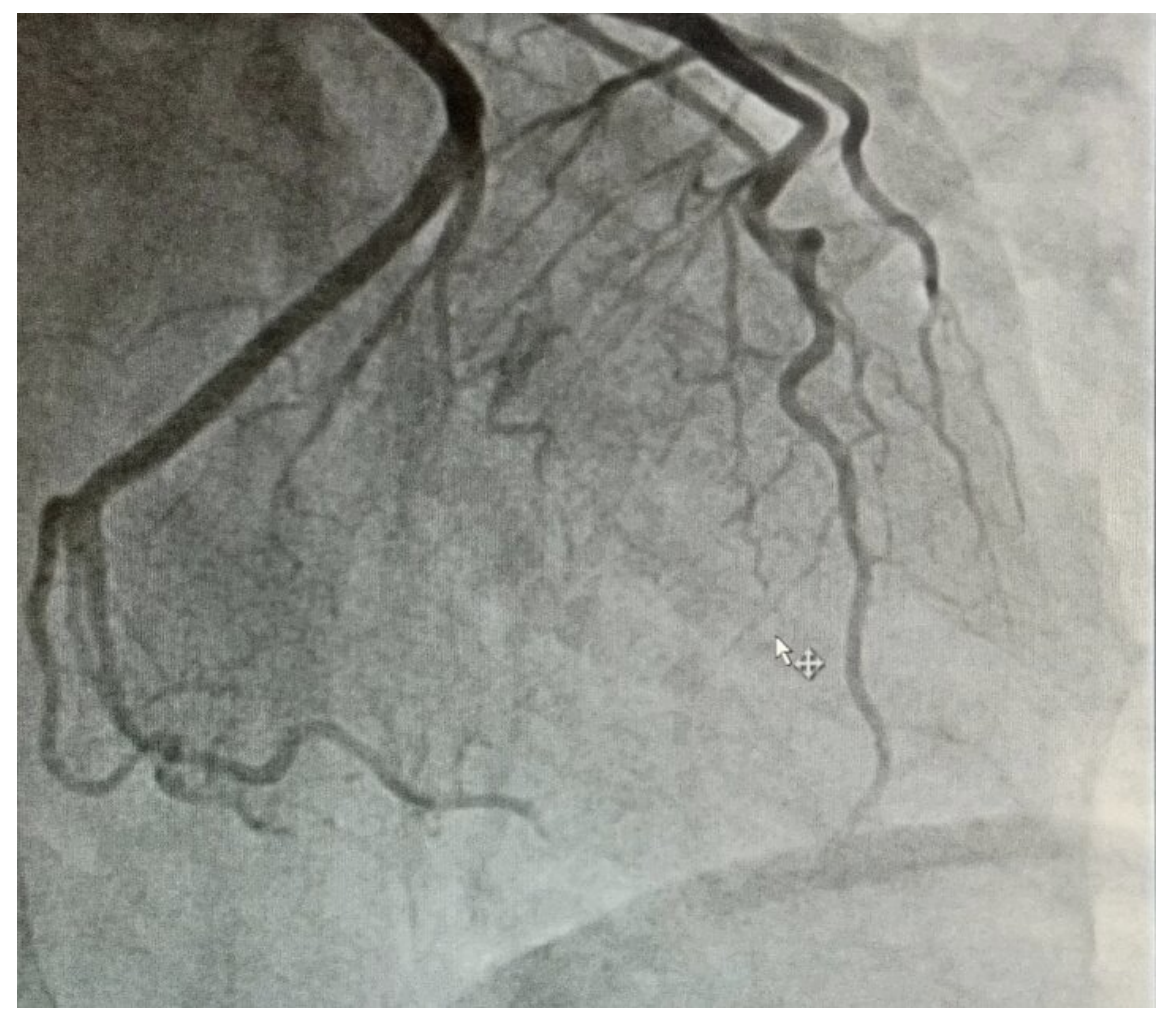




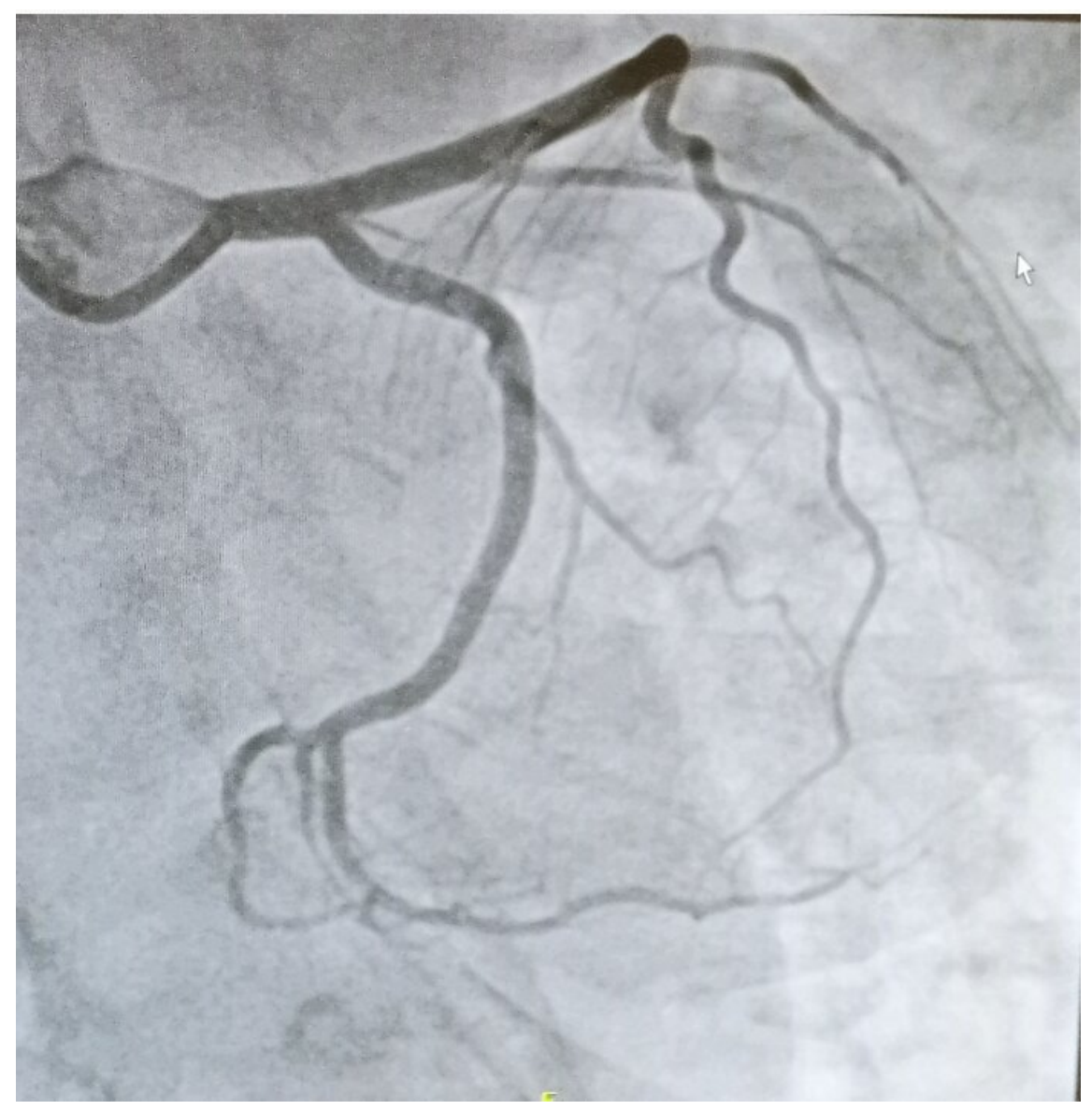




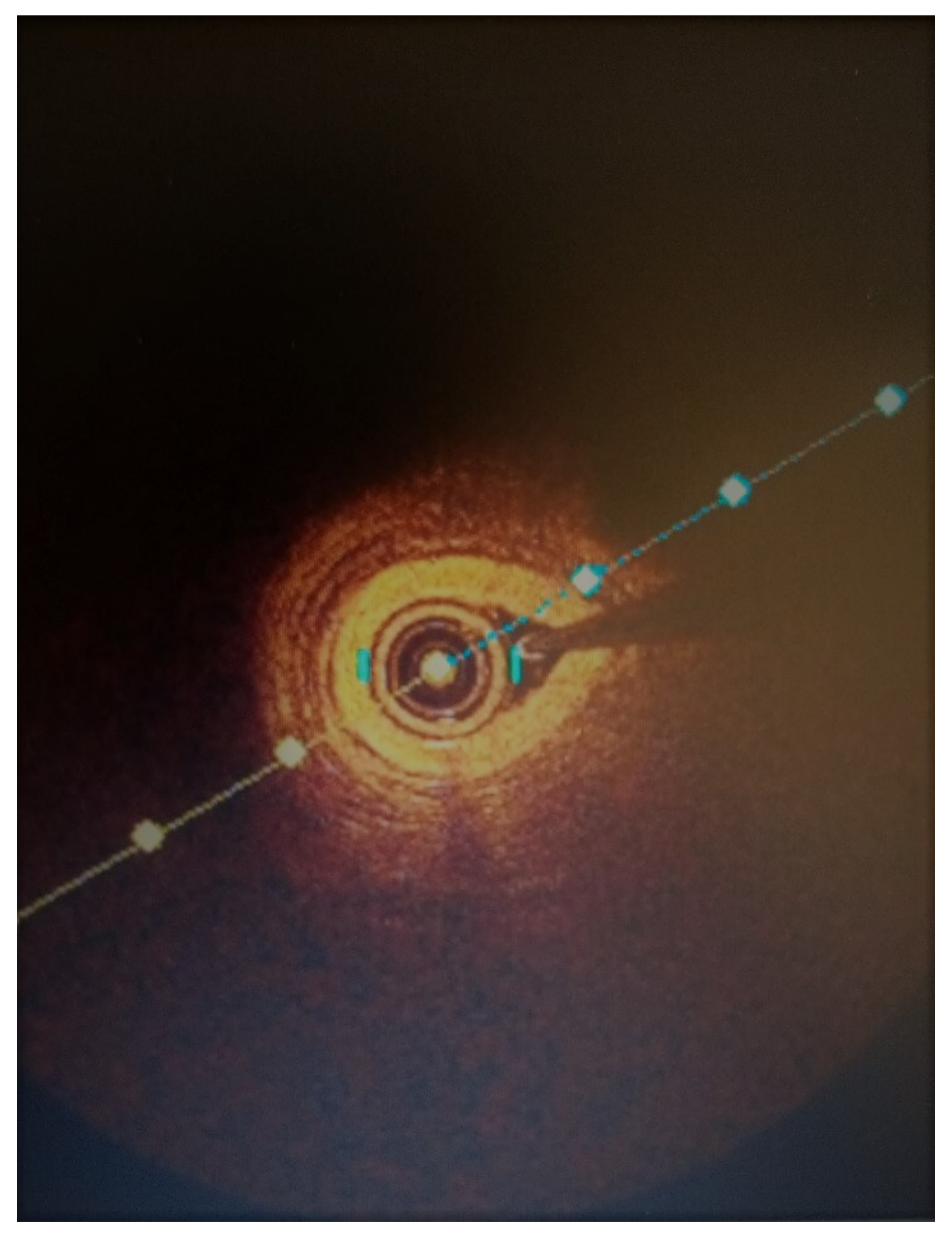

